\title{
A Two-Step Approach for Offset and Position Estimation from Pseudo-Ranges Applied to Multilateration Tracking
}

\author{
Frederik Beutler and Uwe D. Hanebeck \\ Intelligent Sensor-Actuator-Systems Laboratory (ISAS), \\ Institute for Anthropomatics, \\ Karlsruhe Institute of Technology (KIT), Germany. \\ beutler@ieee.org, uwe.hanebeck@ieee.org
}

\begin{abstract}
In multilateration tracking, an object, e.g., an airplane, emits a known reference signal, which is received by several base stations (sensors) located at known positions. The receiving times of the signal at the sensors correspond to the times of arrival (TOA) plus an unknown offset, because the emission time is unknown. Usually, for estimating the position of the object, the receiving times are converted to a larger number of time differences of arrival (TDOA) in order to eliminate the unknown offset. To avoid this conversion, the proposed approach directly uses the receiving times. This is achieved by 1. determining the optimal offset from the redundant measurements in closed form and 2. by considering a modified measurement equation. As a result, position estimation can be performed by optimal stochastic linearization.
\end{abstract}

Keywords: Multilateration Tracking, Localization, Optimal Stochastic Linearization, Gaussian Assumed Density Filter.

\section{Introduction}

In civil air surveillance, secondary radar is used for tracking the trajectory of an aircraft. If the airplane receives a request from a ground station, it send a response, which is then received by several sensors located at known positions. Due to the fact that the receiving signal is known, each sensor can determine the receiving time of the response. The receiving times depend on the times of arrival (TOA), which in turn depend on the distances between the airplane and the sensors, and a time offset. This time offset corresponds to the time, when the aircraft emits the response.

Based on these measured receiving times and the known position of the sensors, the trajectory of the aircraft can be estimated. Usually, the receiving times are converted to time differences of arrival (TDOA) in order to eliminate the unknown emission time. TDOAbased localization approaches are also widely studied in the area of passive speaker localization. There, several closed-form solutions [2, 3, 11, 12] or approaches using a state estimator [10] have been developed.

The proposed approach avoids this conversion, where the unknown emission time is eliminated. Therefore, the new approach directly uses the receiving times for estimation. However, for estimation purposes, the receiving times are converted to pseudo-ranges by multiplying with the wave propagation speed. These pseudoranges are composed of the ranges (distances between object and sensors) and a range offset, which has an equal influence on all ranges. Hence, in the measurement equation the unknown position of the object and an unknown range offset occurs. In order to estimate the position a state estimator is used. Due to nonlinearities in the measurement equation, approximate state estimators have to be used. Popular estimators belong to the class of Gaussian Assumed Density Filters $[5,6,7,8]$, like the Unscented Kalman Filter [7]. This kind of filter assumes that all random variables can be approximated by Gaussian distributions. If the Gaussian assumption is applied, state estimation can be performed efficiently based on the first two moments (mean and covariance). For calculating the required moments, usually sample-based methods are used. On the other hand, for some classes of nonlinear equations like polynomial or trigonometric functions the required moments can be calculated in closed form.

In order to estimate the position of the object and the unknown range offset by using a state estimator two different procedures are suitable based on the assumptions for the range offset. If the assumption holds that the offset has a specific dynamic behavior, the range offset can be augmented in the state vector. In some scenarios, this assumption is not feasible, e.g., when the offset is arbitrary or the dynamic behavior cannot be described by an adequate system model.

The proposed approach assumes that the offset can be arbitrary. For estimation propose the nonlinear measurement equation is first modified in such a way that it is given in polynomial form. Based on this modified 


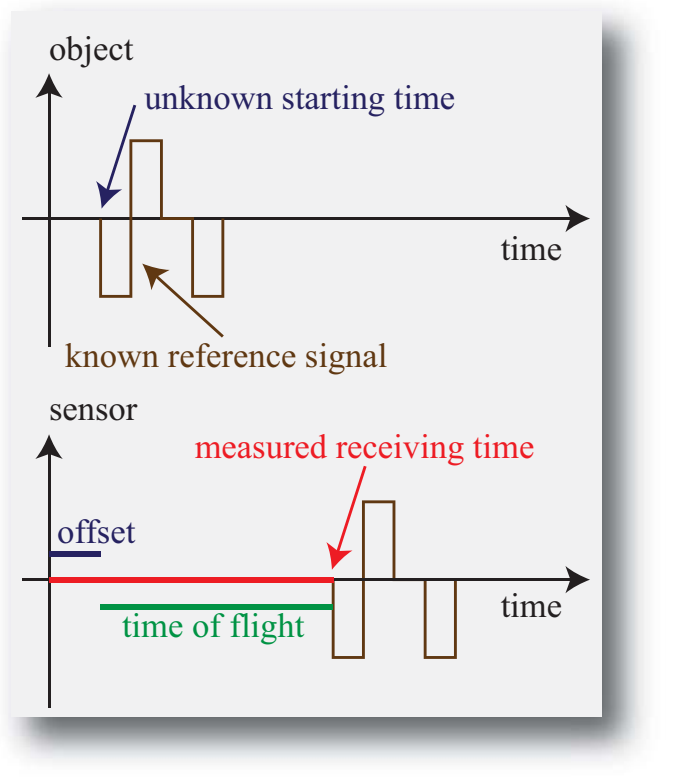

Figure 1: One object emits one known reference signal, which is received by a sensor. The measured receiving time consist of the time of flight and a time offset, because the time when the object emits the signal is unknown.

measurement equation, the range offset and the position are estimated in closed form by using analytic moment calculation. The proposed approach consists of two steps. In the first step, the Mahalanobis distance of the measurement process is minimized in order to calculate the offset. Therefore, no prior knowledge for the offset has to be assumed. In the second step, the position of the object is updated by using the principle of a Gaussian Assumed Density Filter.

The structure of the paper is as follows. In Sec. 2, a problem formulation for multilateration tracking is given. The algorithm is explained in Sec. 3, where the information flow of the proposed approach is described. First, the measurement equation is modified in Sec. 3.1. For estimating the offset and for updating the state, the moments of the measurement process have to be calculated, which is presented in Sec. 3.2. Based on the calculated moments in Sec. 3.3, the algorithm for determining the offset is presented. This estimate for the offset can be used for Bayesian inference, which is explained in Sec. 3.4. The approach is evaluated in simulations in Sec. 4. In Sec. 5, a conclusion is given.

\section{Problem Formulation}

In multilateration tracking, an object, e.g., an aircraft, emits a reference signal, which is detected by several receivers located at known positions. We assume that all receivers are synchronized in such a way that they have the same time base. Furthermore, the time when the object emits the signal is unknown (see

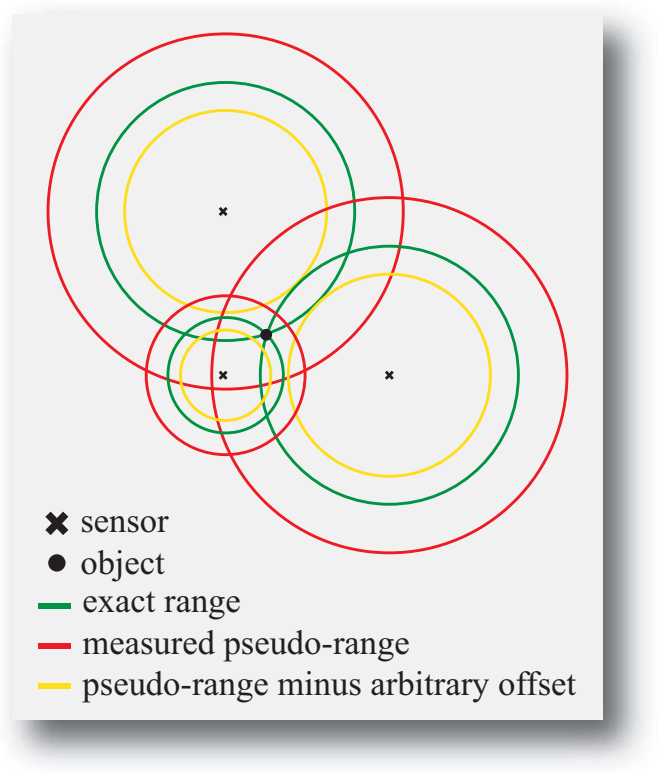

Figure 2: Geometrical interpretation of the problem. The red lines show the measured pseudo-ranges corresponding to measured receiving times. The yellow curves are the ranges subtracted from an arbitrary offset. The green lines show the result if the offset is exactly known. The black dot is the emitter. The black crosses are the receivers.

Fig. 1). This unknown starting time leads to an unknown range offset for all measured pseudo-ranges. In Fig. 2, a geometric interpretation is shown.

The goal of multilateration is to estimate the position of the object based on noisy measured pseudoranges taken at different time steps, in order to track the trajectory of the object.

From a mathematical point of view, a measured pseudo-range $\hat{y}_{i, k}$ is described by a nonlinear measurement equation, which is given by

$$
\boldsymbol{y}_{i, k}=\left\|\underline{S}_{i}-\underline{\boldsymbol{x}}_{k}-\underline{\boldsymbol{v}}_{i, k}\right\|_{2}+b_{k},
$$

where $\underline{S}_{i}$ is the known position from receiver $i, \underline{\boldsymbol{x}}_{k}$ is the position of the object at time step $k, b_{k}$ the unknown range offset, and $\underline{\boldsymbol{v}}_{i, k}$ the noise process, which influences the position [4]. $\hat{y}_{i, k}$ is a realization of the random variable $\boldsymbol{y}_{i, k}$.

Furthermore, the dynamic behavior of the object is described by a system model. This system model comprises assumptions about the possible flight maneuver of the airplane, e.g., position-velocity model [13] or coordinated turn model [1]. In the considered example, we focus on a very simple system model, which is described by

$$
\underline{\boldsymbol{x}}_{k+1}=\underline{\boldsymbol{x}}_{k}+\underline{\boldsymbol{w}}_{k},
$$

where $k$ is the time index and $\underline{\boldsymbol{w}}_{k}$ is zero-mean Gaussian noise characterized by the covariance matrix $\mathbf{C}^{w}$. It 


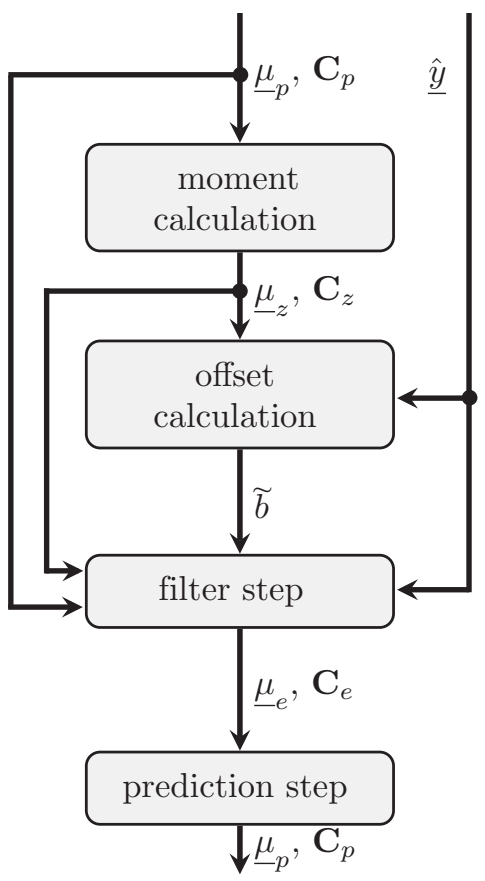

Figure 3: Information flow of the proposed approach.

should be pointed out that the proposed approach is not restricted to the system model in (2).

For the rest of the paper, the time index $k$ is omitted.

\section{Multilateration Tracking}

The proposed approach is a two-step procedure. In a first step, the offset is calculated, which is then used in the second step for updating the position. To calculate the offset $b$ and to update the variable $\underline{\boldsymbol{x}}$, prior knowledge for the random variable $\underline{\boldsymbol{x}}$ in form of a probability density function $f^{p}(\underline{x})$ and realizations $\underline{\hat{y}}$ of the measurement process $\underline{\boldsymbol{y}}$ are used.

To obtain analytic expressions for the algorithm, the measurement equation in (1) is modified and so the mean and covariance of a transformed measurement vector called $\boldsymbol{z}$ can be calculated based on the random variable $\underline{\boldsymbol{x}}$ and a nonlinear function $\underline{h}(\cdot)$ in closed form.

Based on the calculated quantities $\mu^{z}, \mathbf{C}^{z}$ and the actual measurement $\hat{y}$, an estimate $\tilde{b}$ for the unknown offset $b$ is determined by minimizing the Mahalanobis distance.

In the second step, the variable $\underline{\boldsymbol{x}}$ is updated by using Bayesian inference. By applying the Gaussian assumption, which means that the density of the state $f^{p}(\underline{x})$ and the density of the measurement process $f(\underline{z})$ are jointly Gaussian distributed, the parameters of the estimated density $f^{e}(\underline{x})=\mathcal{N}\left(\underline{x}-\mu^{e}, \mathbf{C}^{e}\right)$ is updated.

Furthermore, for recursive estimation a prediction step is used in order to propagate the estimated state to the next time step.

In Fig. 3, the information flow for the proposed approach is shown.

\subsection{Modified Measurement Equation}

The measurement equation (1) for the multilateration problem is now rewritten. First, the offset $b$ is pulled to the left-hand-side of the equation. Then, both sides of the equation are squared in order to eliminate the square root. The new measurement equation is then given in polynomial form by

$$
\begin{aligned}
\left(\boldsymbol{y}_{i}-b\right)^{2} & =\left(\underline{S}_{i}-\underline{\boldsymbol{x}}-\underline{\boldsymbol{v}}_{i}\right)^{\mathrm{T}} \cdot\left(\underline{S}_{i}-\underline{\boldsymbol{x}}-\underline{\boldsymbol{v}}_{i}\right) \\
& =\sum_{m=1}^{M}\left(S_{m, i}-\boldsymbol{x}_{m}-\boldsymbol{v}_{m, i}\right)^{2},
\end{aligned}
$$

where $M$ is the dimension of the considered coordinate system, i.e. in a three-dimensional coordinate system $M=3$. The sum can be written as

$$
\begin{aligned}
& \sum_{m=1}^{M}\left(S_{m, i}-\boldsymbol{x}_{m}-\boldsymbol{v}_{m, i}\right)^{2}= \\
& \underline{1}_{M}^{\mathrm{T}} \cdot\left(\left(\underline{S}_{i}-\underline{\boldsymbol{x}}-\underline{\boldsymbol{v}}_{i}\right) \circ\left(\underline{S}_{i}-\underline{\boldsymbol{x}}-\underline{\boldsymbol{v}}_{i}\right)\right),
\end{aligned}
$$

where $\circ$ is the elementwise product and $\underline{1}_{M}$ is a one vector with length $M$. Furthermore, a new random variable $\boldsymbol{z}_{i}:=\left(\boldsymbol{y}_{i}-b\right)^{2}$ is introduced. For this new random variable, the measurement equation is given by

$$
\boldsymbol{z}_{i}=\underline{1}_{M}^{\mathrm{T}} \cdot\left(\left(\underline{S}_{i}-\underline{\boldsymbol{x}}-\underline{\boldsymbol{v}}_{i}\right) \circ\left(\underline{S}_{i}-\underline{\boldsymbol{x}}-\underline{\boldsymbol{v}}_{i}\right)\right) .
$$

The resulting measurement equation for $N$ receivers is described by

$$
\begin{aligned}
\underline{\boldsymbol{z}} & =\left[\begin{array}{c}
\underline{1}_{M}^{\mathrm{T}} \cdot\left(\left(\underline{S}_{1}-\underline{\boldsymbol{x}}-\underline{\boldsymbol{v}}_{1}\right) \circ\left(\underline{S}_{1}-\underline{\boldsymbol{x}}-\underline{\boldsymbol{v}}_{1}\right)\right) \\
\vdots \\
\underline{1}_{M}^{\mathrm{T}} \cdot\left(\left(\underline{S}_{N}-\underline{\boldsymbol{x}}-\underline{\boldsymbol{v}}_{N}\right) \circ\left(\underline{S}_{N}-\underline{\boldsymbol{x}}-\underline{\boldsymbol{v}}_{N}\right)\right)
\end{array}\right] \\
& =\underline{h}(\underline{\boldsymbol{x}}, \underline{\boldsymbol{v}}) .
\end{aligned}
$$

Based on the modified measurement equation (4), the required moments (mean and covariance) of the random variable $\underline{z}$ can be specified analytically.

\subsection{Moment Calculation}

For the offset calculation and the filter step, the moments $\mu^{z}$ and $\mathbf{C}^{z}$ are required. The required moments are calculated by analytic moment calculation [9]. In order to calculate the required moments in closed form, the densities for the random variables $\underline{x}$ and $\underline{\boldsymbol{v}}$ are assumed to be Gaussian distributed, given by $\mathcal{N}\left(\underline{x}-\underline{\mu}^{p}, \mathbf{C}^{p}\right)$ and $\mathcal{N}\left(\underline{v}, \mathbf{C}^{v}\right)$, where $\mu^{p}$ and $\mathbf{C}^{p}$ describe the predicted mean and covariance, respectively. The random variable $\underline{\boldsymbol{v}}$ is zero-mean, with a covariance given by

$$
\mathbf{C}^{v}=\left[\begin{array}{ccccc}
\mathbf{C}_{1}^{v} & \ldots & \mathbf{C}_{1, j}^{v} & \ldots & \mathbf{C}_{1, N}^{v} \\
\vdots & \vdots & \vdots & \vdots & \vdots \\
\mathbf{C}_{i, 1}^{v} & \ldots & \mathbf{C}_{i, j}^{v} & \ldots & \mathbf{C}_{i, N}^{v} \\
\vdots & \vdots & \vdots & \vdots & \vdots \\
\mathbf{C}_{N, 1}^{v} & \ldots & \mathbf{C}_{N, j}^{v} & \ldots & \mathbf{C}_{N}^{v}
\end{array}\right]
$$


where $\mathbf{C}_{i, j}^{v}$ describes the covariance between the senor $i$ and sensor $j$. In order to have a comprehensive derivation of the moment calculation, a new random variable

$$
\underline{\boldsymbol{f}}=\left[\begin{array}{c}
\left(\underline{S}_{1}-\underline{\boldsymbol{x}}-\underline{\boldsymbol{v}}_{1}\right) \\
\vdots \\
\left(\underline{S}_{N}-\underline{\boldsymbol{x}}-\underline{\boldsymbol{v}}_{N}\right)
\end{array}\right]
$$

is used, which consists of $N \cdot M$ entries. The variable $N$ is the number of sensors and $M$ corresponds to the dimension of the considered coordinate system. The measurement equation (4) is then given by

$$
\underline{\boldsymbol{z}}=\underbrace{\left[\begin{array}{ccc}
\underline{1}_{M}^{\mathrm{T}} & \underline{0}_{N}^{\mathrm{T}} \cdot M-M & \\
\underline{0}_{M}^{\mathrm{T}} & \underline{1}_{M}^{\mathrm{T}} & \underline{0}_{N \cdot M-2 M}^{\mathrm{T}} \\
\vdots & \vdots & \vdots \\
\underline{0}_{N \cdot M-M}^{\mathrm{T}} & & \underline{1}_{M}^{\mathrm{T}}
\end{array}\right]}_{\mathbf{K}^{\mathrm{T}}} \cdot\left(\underline{\boldsymbol{f}}^{\mathrm{T}} \underline{\boldsymbol{f}}\right),(6)
$$

where first each entry of the vector $\boldsymbol{f}$ is squared. Then, parts of the resulting vector are accumulate according to (3) by multiplying with the matrix $\mathbf{K}^{\mathrm{T}}$. Furthermore, the matrix $\mathbf{K}$ is given in short form $\mathbf{K}=$ $\mathbf{I}_{N, N} \otimes \underline{1}_{M}, \mathbf{I}$ is the identity matrix, $\otimes$ is the Kronecker product, $\underline{1}_{M}$ is a one vector with length $M, \underline{0}_{M}$ is a zero vector with length $M$, and o the elementwise product. Due to the fact that (5) describes a linear relationship, the density of the random variable $\boldsymbol{f}$ is Gaussian distributed $\mathcal{N}\left(\underline{f}-\underline{\mu}^{f}, \mathbf{C}^{f}\right)$, with mean and covariance

$$
\underline{\mu}^{f}=\left[\begin{array}{c}
\underline{S}_{1}-\underline{\mu}^{p} \\
\vdots \\
\underline{S}_{N}-\underline{\mu}^{p}
\end{array}\right], \mathbf{C}^{f}=\mathbf{C}^{v}+\mathbf{1}_{N, N} \otimes \mathbf{C}^{p} .
$$

In the matrix $\mathbf{1}_{N, N}=\underline{1}_{N} \cdot\left(\underline{1}_{N}\right)^{\mathrm{T}}$, all entries are equal to one.

\subsubsection{Mean $\mu^{z}$}

The mean $\underline{\mu}^{z}$ of the random variable $\underline{z}$ is defined by

$$
\underline{\mu}^{z}=\mathrm{E}_{\underline{z}}\{\underline{z}\}=\int \underline{z} \cdot f(\underline{z}) \mathrm{d} \underline{z},
$$

which it can be expanded to

$$
\underline{\mu}^{z}=\iiint \underline{z} \cdot f(\underline{z}, \underline{x}, \underline{v}) \mathrm{d} \underline{z} \mathrm{~d} \underline{x} \mathrm{~d} \underline{v} .
$$

The joint density $f(\underline{z}, \underline{x}, \underline{v})$ of the random variables can be replaced by using Baye's rule

$$
\begin{aligned}
f(\underline{z}, \underline{x}, \underline{v}) & =f(\underline{z} \mid \underline{x}, \underline{v}) \cdot f(\underline{x}, \underline{v}) \\
& =\delta(\underline{z}-\underline{h}(\underline{x}, \underline{v})) \cdot f(\underline{x}, \underline{v}) .
\end{aligned}
$$

Furthermore, the random variables $\underline{\boldsymbol{x}}$ and $\underline{\boldsymbol{v}}$ are assumed as independent, so that the joint density $f(\underline{x}, \underline{v})$ can be replaced by

$$
f(\underline{x}, \underline{v})=f^{p}(\underline{x}) \cdot f(\underline{v}) .
$$

By using the sifting property of the Dirac delta distribution the mean $\mu^{z}$ is calculated according to

$$
\begin{aligned}
\underline{\mu}^{z} & =\iint \underline{h}(\underline{x}, \underline{v}) f^{p}(\underline{x}) \cdot f(\underline{v}) \mathrm{d} \underline{x} \mathrm{~d} \underline{v} \\
& =\mathrm{E}_{\underline{\boldsymbol{x}}, \underline{\boldsymbol{v}}}\{\underline{h}(\underline{\boldsymbol{x}}, \underline{\boldsymbol{v}})\} .
\end{aligned}
$$

For calculating the mean $\underline{\mu}^{z}$, (4) is used in (7). By using the new random variable $\underline{f}$ and (6), the mean $\underline{\mu}^{z}$ is given by

$$
\underline{\mu^{z}}=\mathrm{E}_{\underline{\boldsymbol{f}}}\left\{\mathbf{K}^{\mathrm{T}} \cdot(\underline{\boldsymbol{f}} \circ \underline{\boldsymbol{f}})\right\}=\mathbf{K}^{\mathrm{T}} \cdot \mathrm{E}_{\underline{\boldsymbol{f}}}\{\underline{\boldsymbol{f}} \circ \underline{\boldsymbol{f}}\},
$$

where the linear property of the expectation operator is used. Due to the elementwise operator $\circ$ in the expectation operator, the result is the second-order noncentral moment of each entry from the random variable $\underline{\boldsymbol{f}}$ according to

$$
\mathrm{E}_{\boldsymbol{f}_{i}}\left\{\left(\boldsymbol{f}_{i}\right)^{2}\right\}=\left(\mu_{i}^{f}\right)^{2}+C_{i, i}^{f}
$$

where $\mu_{i}^{f}$ is the $i$ th entry of the vector $\underline{\mu}^{f}$ and $C_{i, i}^{f}$ is the $i, i$ th entry of the matrix $\mathbf{C}^{f}$ for $i=1, \ldots, N \cdot M$. If this is generalize to all entries the square $\left(\mu_{i}^{f}\right)^{2}$ becomes the elementwise product of $\underline{\mu}^{f} \circ \underline{\mu}^{f}$ and $C_{i, i}^{f}$ becomes a vector consisting of the diagonal entries of the matrix $\mathbf{C}^{f}$, which results for the mean $\underline{\mu}^{z}$ then in

$$
\underline{\mu}^{z}=\mathbf{K}^{\mathrm{T}} \cdot\left(\underline{\mu}^{f} \circ \underline{\mu}^{f}+\operatorname{diag}\left(\mathbf{C}^{f}\right)\right) .
$$

\subsubsection{Covariance $\mathrm{C}^{z}$}

In analogy, the covariance $\mathbf{C}^{z}$ is calculated as

$$
\begin{aligned}
\mathbf{C}^{z}= & \iint\left(\underline{h}(\underline{x}, \underline{v})-\underline{\mu}^{z}\right) \cdot \\
& \left(\underline{h}(\underline{x}, \underline{v})-\underline{\mu}^{z}\right)^{\mathrm{T}} \cdot f^{p}(\underline{x}) \cdot f(\underline{v}) \mathrm{d} \underline{x} \mathrm{~d} \underline{v} \\
= & \mathrm{E}_{\underline{\boldsymbol{x}}, \underline{v}}\left\{\underline{h}(\underline{\boldsymbol{x}}, \underline{\boldsymbol{v}}) \cdot \underline{h}(\underline{\boldsymbol{x}}, \underline{\boldsymbol{v}})^{\mathrm{T}}\right\}-\underline{\mu}^{z} \cdot\left(\underline{\mu}^{z}\right)^{\mathrm{T}} .
\end{aligned}
$$

For determining the covariance (4) is used in (8). By using the random variable $\boldsymbol{f}$ and (6), the covariance $\mathbf{C}^{z}$ is given by

$$
\begin{aligned}
\mathbf{C}^{z} & =\mathrm{E}_{\underline{\boldsymbol{f}}}\left\{\mathbf{K}^{\mathrm{T}} \cdot(\underline{\boldsymbol{f}} \circ \underline{\boldsymbol{f}}) \cdot\left(\mathbf{K}^{\mathrm{T}} \cdot(\underline{\boldsymbol{f}} \circ \underline{\boldsymbol{f}})\right)^{\mathrm{T}}\right\}-\underline{\mu}^{z}\left(\underline{\mu}^{z}\right)^{\mathrm{T}} \\
& =\mathbf{K}^{\mathrm{T}} \cdot \mathrm{E}_{\underline{\boldsymbol{f}}}\left\{(\underline{\boldsymbol{f}} \circ \underline{\boldsymbol{f}}) \cdot\left(\underline{\boldsymbol{f}}^{\mathrm{T}} \circ \underline{\boldsymbol{f}}^{\mathrm{T}}\right)\right\} \cdot \mathbf{K}-\underline{\mu}^{z}\left(\underline{\mu}^{z}\right)^{\mathrm{T}} .
\end{aligned}
$$

In order to determine the covariance $\mathbf{C}^{z}$ the expected value $\mathrm{E}_{\underline{\boldsymbol{f}}}\left\{(\underline{\boldsymbol{f}} \circ \underline{\boldsymbol{f}}) \cdot\left(\underline{\boldsymbol{f}}^{\mathrm{T}} \circ \underline{\boldsymbol{f}}^{\mathrm{T}}\right)\right\}$ has to been calculated. The Cartesian product of the squared variables can be rewritten as the squared Cartesian product of the variables, which is given by

$$
\mathbf{C}^{z}=\mathbf{K}^{\mathrm{T}} \cdot \mathrm{E}_{\underline{\boldsymbol{f}}}\left\{\left(\underline{\boldsymbol{f}} \cdot \underline{\boldsymbol{f}}^{\mathrm{T}}\right) \circ\left(\underline{\boldsymbol{f}} \cdot \underline{\boldsymbol{f}}^{\mathrm{T}}\right)\right\} \cdot \mathbf{K}-\underline{\mu}^{z}\left(\underline{\mu}^{z}\right)^{\mathrm{T}} .
$$


For determining the expected value $\mathrm{E}_{\boldsymbol{f}}\left\{\left(\underline{\boldsymbol{f}} \cdot \underline{\boldsymbol{f}}^{\mathrm{T}}\right) \circ\left(\underline{\boldsymbol{f}} \cdot \underline{\boldsymbol{f}}^{\mathrm{T}}\right)\right\}$ of the matrix, each entry can be calculated separately due to the linearity of the expectation operator. The $i, j$ th entry of the matrix is the fourth-order non-central moment, which is given by

$$
\begin{aligned}
& \mathrm{E}_{\boldsymbol{f}_{i}, \boldsymbol{f}_{j}}\left\{\left(\boldsymbol{f}_{i}\right)^{2} \cdot\left(\boldsymbol{f}_{j}\right)^{2}\right\}= \\
&\left(\left(\mu_{i}^{f}\right)^{2}+C_{i, i}^{f}\right) \cdot\left(\left(\mu_{j}^{f}\right)^{2}+C_{j, j}^{f}\right) \\
&+4 \cdot \mu_{i}^{f} \cdot \mu_{j}^{f} \cdot C_{i, j}^{f}+2 \cdot\left(C_{i, j}^{f}\right)^{2},
\end{aligned}
$$

where $\mu_{i}^{f}$ is the $i$ th entry of the vector $\underline{\mu}^{f}$ and $C_{i, j}^{f}$ is the $i, j$ th entry of the matrix $\mathbf{C}^{f}$ for $i=1, \ldots, N \cdot M$ and $j=1, \ldots, N \cdot M$. If this is generalized to all entries to the matrix, the resulting matrix can be written as

$$
\begin{aligned}
& \mathrm{E}_{\boldsymbol{f}}\left\{\left(\underline{\boldsymbol{f}} \cdot \underline{\boldsymbol{f}}^{\mathrm{T}}\right) \circ\left(\underline{\boldsymbol{f}} \cdot \underline{\boldsymbol{f}}^{\mathrm{T}}\right)\right\}= \\
& \left(\underline{\mu}^{f} \circ \underline{\mu}^{f}+\operatorname{diag}\left(\mathbf{C}^{f}\right)\right) \cdot\left(\underline{\mu}^{f} \circ \underline{\mu}^{f}+\operatorname{diag}\left(\mathbf{C}^{f}\right)\right)^{\mathrm{T}} \\
& +4 \cdot\left(\underline{\mu}^{f} \cdot\left(\underline{\mu}^{f}\right)^{\mathrm{T}}\right) \circ \mathbf{C}^{f}+2 \cdot \mathbf{C}^{f} \circ \mathbf{C}^{f} .
\end{aligned}
$$

Using the result of (10) in (9), the covariance $\mathbf{C}^{z}$ is given by

$$
\mathbf{C}^{z}=\mathbf{K}^{\mathrm{T}} \cdot\left(4 \cdot\left(\underline{\mu}^{f} \cdot\left(\underline{\mu}^{f}\right)^{\mathrm{T}}\right) \circ \mathbf{C}^{f}+2 \cdot \mathbf{C}^{f} \circ \mathbf{C}^{f}\right) \cdot \mathbf{K},
$$

where the Cartesian product $\underline{\mu}^{z} \cdot\left(\underline{\mu}^{z}\right)^{\mathrm{T}}$ is given by

$$
\begin{aligned}
\underline{\mu}^{z} \cdot\left(\underline{\mu}^{z}\right)^{\mathrm{T}}= & \mathbf{K}^{\mathrm{T}} \cdot\left(\underline{\mu}^{f} \circ \underline{\mu}^{f}+\operatorname{diag}\left(\mathbf{C}^{f}\right)\right) . \\
& \left(\underline{\mu}^{f} \circ \underline{\mu}^{f}+\operatorname{diag}\left(\mathbf{C}^{f}\right)\right)^{\mathrm{T}} \cdot \mathbf{K} .
\end{aligned}
$$

\subsection{Offset Calculation}

For determining an estimate $\tilde{b}$ for the unknown offset $b$, the Mahalanobis distance is minimized. As the square root is a monotonic function, this is equivalent to minimizing the squared Mahalanobis distance given by

$$
\begin{aligned}
M(b)= & \left(\underline{\mu}^{z}-(\underline{\hat{y}}-\underline{1} \cdot b) \circ(\underline{\hat{y}}-\underline{1} \cdot b)\right)^{\mathrm{T}} \cdot\left(\mathbf{C}^{z}\right)^{-1} . \\
& \left(\underline{\mu}^{z}-(\underline{\hat{y}}-\underline{1} \cdot b) \circ(\underline{\hat{y}}-\underline{1} \cdot b)\right),
\end{aligned}
$$

which leads to a simpler optimization problem. For finding the minimum the derivative with respect to the parameter $b$ must be zero according to

$$
\begin{aligned}
\frac{\mathrm{d} M}{\mathrm{~d} b} & =4(\underline{\hat{y}}-\underline{1} b)^{\mathrm{T}}\left(\mathbf{C}^{z}\right)^{-1}\left(\underline{\mu}^{z}-(\underline{\hat{y}}-\underline{1} b) \circ(\underline{\hat{y}}-\underline{1} b)\right) \\
& =0 .
\end{aligned}
$$

This equation can be rewritten as a polynomial function according to

$$
b^{3} \cdot a_{1}+b^{2} \cdot a_{2}+b \cdot a_{3}+a_{4}=0,
$$

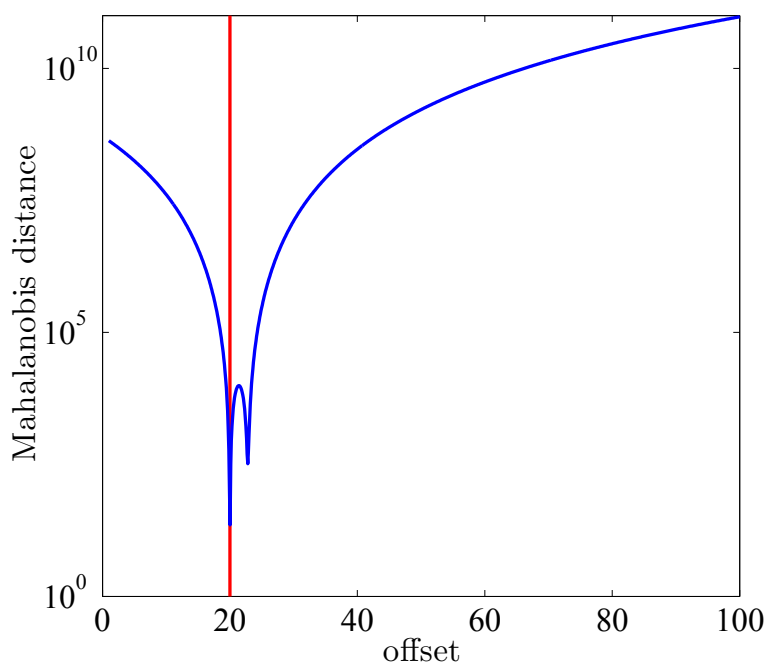

Figure 4: Mahalanobis distance evaluated for different offset values. The red line shows the true offset.

where the factors $a_{1}, \ldots, a_{4}$ are

$$
\begin{aligned}
a_{1}= & \underline{1}^{\mathrm{T}} \cdot\left(\mathbf{C}^{z}\right)^{-1} \cdot \underline{1}, \\
a_{2}= & -3 \cdot \underline{1}^{\mathrm{T}} \cdot\left(\mathbf{C}^{z}\right)^{-1} \cdot \underline{\hat{y}}, \\
a_{3}= & -\underline{1}^{\mathrm{T}} \cdot\left(\mathbf{C}^{z}\right)^{-1} \cdot \underline{\mu}^{z}+\underline{1}^{\mathrm{T}} \cdot\left(\mathbf{C}^{z}\right)^{-1} \cdot(\underline{\hat{y}} \circ \underline{\hat{y}}) \\
& +2 \cdot(\underline{\hat{y}})^{\mathrm{T}} \cdot\left(\mathbf{C}^{z}\right)^{-1} \cdot(\underline{\hat{y}}), \\
a_{4}= & (\underline{\hat{y}})^{\mathrm{T}} \cdot\left(\mathbf{C}^{z}\right)^{-1} \cdot \underline{\mu}^{z}-(\underline{\hat{y}})^{\mathrm{T}} \cdot\left(\mathbf{C}^{z}\right)^{-1} \cdot(\underline{\hat{y}} \circ \underline{\hat{y}}) .
\end{aligned}
$$

First, the three roots $b_{1}, b_{2}$, and $b_{3}$ of the polynomial function (11) are determined, where $b_{1}<b_{2}<b_{3}$. If these roots are used for evaluating the distance $M(b)$ (see Fig. 4), the smallest $b_{1}$ and largest $b_{3}$ value for the roots provides each a local minimum and the remaining value $b_{2}$ for the third root a local maximum. Furthermore, the smallest root $b_{1}$ corresponds to the estimated offset $b_{1}=\tilde{b}$, because the largest root $b_{3}$ provides negative ranges, i.e. in this case $\left(\hat{y}-\underline{1}_{3}\right)<\underline{0}$ and so the ranges are $\underline{h}(\underline{\boldsymbol{x}}, \underline{\boldsymbol{v}})<\underline{0}$, so the root $b_{3}$ can be omitted.

\section{$3.4 \quad$ Filter Step}

In the filter step, the predicted density $f^{p}(\underline{x})$ is updated based on the measurement $\hat{y}$. As the result of the filter step, the estimated density $f^{e}(\underline{x})=$ $\mathcal{N}\left(\underline{x}-\mu^{e}, \mathbf{C}^{e}\right)$ is provided. Thanks to the additional Gaussian assumption, the estimated mean $\mu^{e}$ and covariance $\mathbf{C}^{e}$ are updated according to

$$
\begin{aligned}
\mu^{e} & =\mu^{p}+\mathbf{C}^{x, z} \cdot\left(\mathbf{C}^{z}\right)^{-1} \cdot\left((\underline{\hat{y}}-\underline{1} \tilde{b}) \circ(\underline{\hat{y}}-\underline{1} \tilde{b})-\underline{\mu}^{z}\right), \\
\mathbf{C}^{e} & =\mathbf{C}^{p}-\mathbf{C}^{x, z} \cdot\left(\mathbf{C}^{z}\right)^{-1} \cdot\left(\mathbf{C}^{x, z}\right)^{\mathrm{T}},
\end{aligned}
$$

where the estimated offset $\tilde{b}$ has to be subtracted from the measurement $\underline{\hat{y}}$ and this result has to be squared. 


\subsubsection{Cross-Covariance $\mathbf{C}^{x, z}$}

Furthermore, the cross-covariance $\mathbf{C}^{x, z}$ is calculated as

$$
\begin{aligned}
& \mathbf{C}^{x, z}= \\
& \iint\left(\underline{x}-\underline{\mu}^{p}\right) \cdot\left(\underline{h}(\underline{x}, \underline{v})-\underline{\mu}^{z}\right)^{\mathrm{T}} \cdot f^{p}(\underline{x}) \cdot f(\underline{v}) \mathrm{d} \underline{x} \mathrm{~d} \underline{v} \\
& =\mathrm{E}_{\underline{\boldsymbol{x}}, \underline{\boldsymbol{v}}}\left\{\underline{\boldsymbol{x}} \cdot \underline{h}(\underline{\boldsymbol{x}}, \underline{\boldsymbol{v}})^{\mathrm{T}}\right\}-\underline{\mu}^{p} \cdot\left(\underline{\mu}^{z}\right)^{\mathrm{T}} .
\end{aligned}
$$

By using (4), the expected value $\mathrm{E}_{\underline{x}, \underline{v}}\{\cdot\}$ is

$$
\begin{aligned}
& \mathrm{E}_{\underline{\boldsymbol{x}}, \underline{\boldsymbol{v}}}\left\{\underline{\boldsymbol{x}} \cdot \underline{h}(\underline{\boldsymbol{x}}, \underline{\boldsymbol{v}})^{\mathrm{T}}\right\}= \\
& -2 \mathbf{C}^{p} \cdot\left[\underline{S}_{1}-\underline{\mu}^{p} \quad \cdots \quad \underline{S}_{N}-\underline{\mu}^{p}\right]+\underline{\mu}^{p} \cdot\left(\underline{\mu}^{z}\right)^{\mathrm{T}}
\end{aligned}
$$

and the resulting cross-covariance $\mathbf{C}^{x, z}$ is then given by

$$
\mathbf{C}^{x, z}=-2 \mathbf{C}^{p} \cdot\left[\begin{array}{lll}
\underline{S}_{1}-\underline{\mu}^{p} & \cdots & \underline{S}_{N}-\underline{\mu}^{p}
\end{array}\right] .
$$

\subsection{Prediction Step}

For recursive estimation, the system model in (2) is used. The prediction is performed by using the Kalman Filter prediction equation for the predicted mean and covariance

$$
\underline{\mu}^{p}=\underline{\mu}^{e}, \mathbf{C}^{p}=\mathbf{C}^{e}+\mathbf{C}^{w} .
$$

If a different motion model is used, e.g., a positionvelocity model [13], only the cross-covariance has to be considered as the mean $\mu^{z}$ and covariance $\mathbf{C}^{z}$ only depends on the position and not on the velocity, i.e., additionally the cross-covariance for the filter step depending on the velocity has to be calculated.

\section{Simulation Results}

In the simulation, the problem of multilateration tracking is addressed. The proposed approach is compared to two classic approaches. In the first approach, ranges are measured, which corresponds to a TOAbased approach. In this case, the measurement equation is given by

$$
\boldsymbol{y}_{i}=\left\|\underline{S}_{i}-\underline{\boldsymbol{x}}-\underline{\boldsymbol{v}}_{i}\right\|_{2},
$$

where $i=1, \ldots, N$ and the offset is precisely known. The TOA-based approach is the so called trilateration, where spheres have to be intersected.

In the second approach, range differences (TDOAbased) are used for estimation. By using (1), the measurement equation for the second case is given by

$$
\underbrace{\boldsymbol{y}_{1}-\boldsymbol{y}_{i}}_{\Delta \boldsymbol{y}_{i-1}}=\left\|\underline{S}_{1}-\underline{\boldsymbol{x}}-\underline{\boldsymbol{v}}_{1}\right\|_{2}-\left\|\underline{S}_{i}-\underline{\boldsymbol{x}}-\underline{\boldsymbol{v}}_{i}\right\|_{2},
$$

where $i=2, \ldots, N$. In the TDOA-based approach sensor 1 is selected as the reference sensor. By subtracting the measurements at the sensors $i=2, \ldots, N$ from

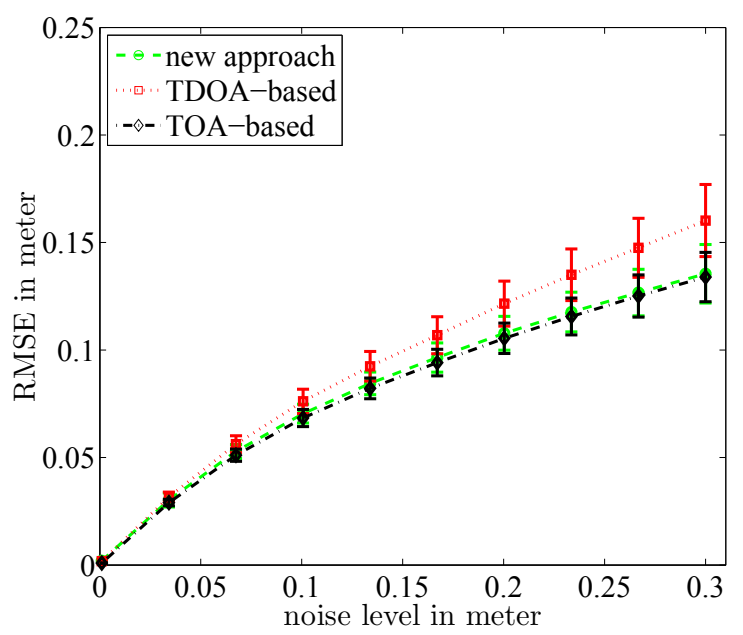

(a) Mean and standard deviation of the RMSE for the three approaches. The black line is the result, when the offset is exactly known. The green line show the result of the proposed approach. The result of the TDOA-based approach is shown in the red line.

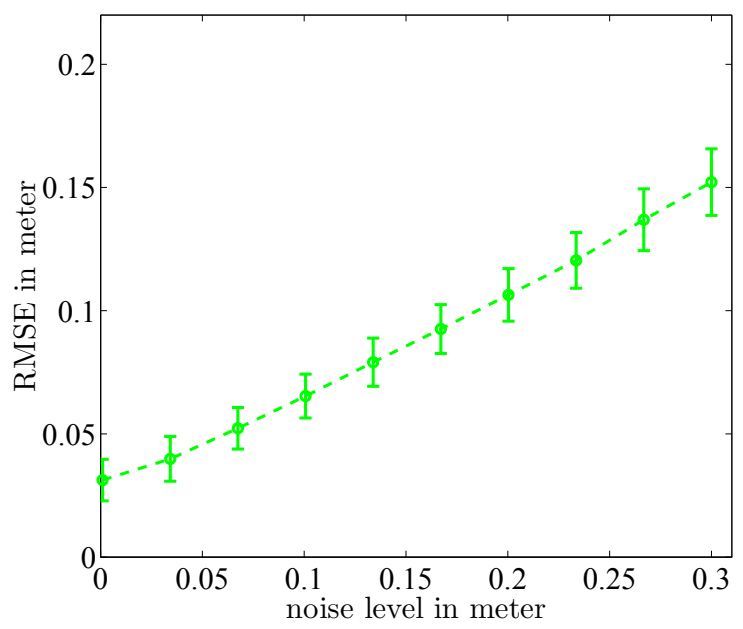

(b) Mean and standard deviation of the RMSE for the offset.

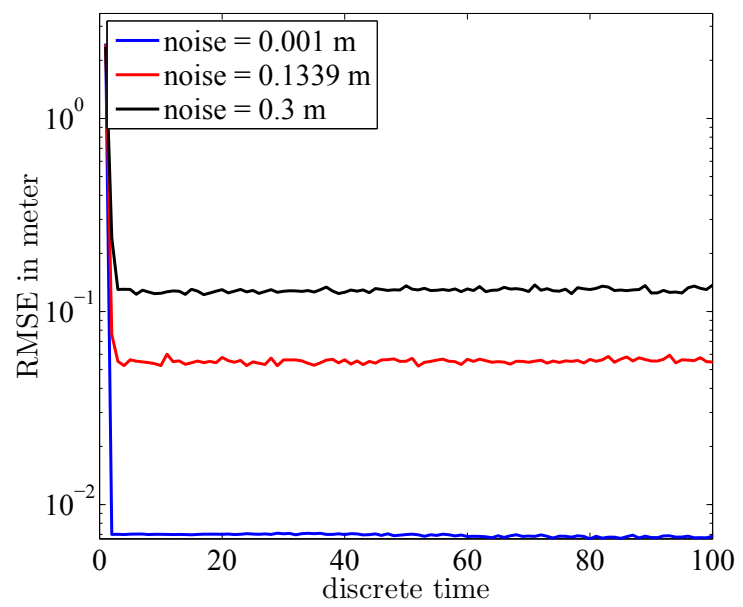

(c) Mean of the RMSE of the Offset at different noise level for all trajectories.

Figure 5: Results of the three estimators by using four sensors. 
the measurement at sensor 1 the measured range differences $\Delta \boldsymbol{y}_{i-1}$ are provided. Hence, The TDOA-based approach relies on intersection of hyperboloids.

At different noise level ranging from 0.001 meter to 0.3 meter, 1000 random trajectories are generated. Each trajectory consists of 100 measurement points. The position of the emitter is estimated based on the measurements at the four sensors, which are located at the positions

$$
\begin{aligned}
& \underline{S}_{1}=\left[\begin{array}{l}
-1 \\
-1
\end{array}\right] \mathrm{m}, \underline{S}_{2}=\left[\begin{array}{l}
-1 \\
+1
\end{array}\right] \mathrm{m}, \\
& \underline{S}_{3}=\left[\begin{array}{l}
+1 \\
-1
\end{array}\right] \mathrm{m}, \underline{S}_{4}=\left[\begin{array}{l}
+1 \\
+1
\end{array}\right] \mathrm{m} .
\end{aligned}
$$

Furthermore, the simulated range offset is a realization of a uniform distribution ranging from 0 meter to 100 meter. For every measurement at one time step, a new offset is generated.

The three estimators are initialized with zero mean and an initial covariance $\operatorname{diag}\left(\left[\begin{array}{ll}10 \mathrm{~m}^{2} & 10 \mathrm{~m}^{2}\end{array}\right]\right)$. The chosen system model for the three estimators is given by (2), where the covariance for the process noise is $\mathbf{C}^{w}=\operatorname{diag}\left(\left[\begin{array}{ll}0.01 \mathrm{~m}^{2} & 0.01 \mathrm{~m}^{2}\end{array}\right]\right)$. The estimator in the TOA-based scenario relies on optimal stochastic linearization, where the required moments for the filter step are calculated by analytic moment calculation as is shown in Sec. 3.2 and Sec. 3.4. In the TDOA-based scenario, the UKF is used for estimation.

The average of the root-mean-square-error (RMSE) and its standard deviation from the three approaches is shown in Fig. 5 (a), where the black line is the lower bound for the considered problem. Thus, the offset is exactly known in this case. Compared to the green line, when the offset is estimated, the average of the RMSE of the proposed approach is slightly higher. The red line shows the results for the TDOA-based approach. For small measurement noise, this approach provides satisfactory results, but if the measurement noise increases, the accuracy of the TDOA-based approach decreases significantly compare to the new and the TOA-based approach.

Furthermore, the RMSE of the estimated offset increases approximately linear with the noise level as is shown in Fig. 5 (b). The average RMSE over the test runs for three different noise levels is shown in Fig. 5 (c). The transition time for the offset is three time steps. After that, the average of the RMSE for the offset is constant.

In the next simulation the number of sensors is decreased (three sensors). The accuracy is decreasing and the deviation between the three approaches is increasing (see Fig. 6 (a)). Furthermore, the average of the RMSE for the offset increases (see Fig. 6 (b)). As in the scenario before, the transition time for calculating the offset is three time steps (see Fig. 6 (c)).

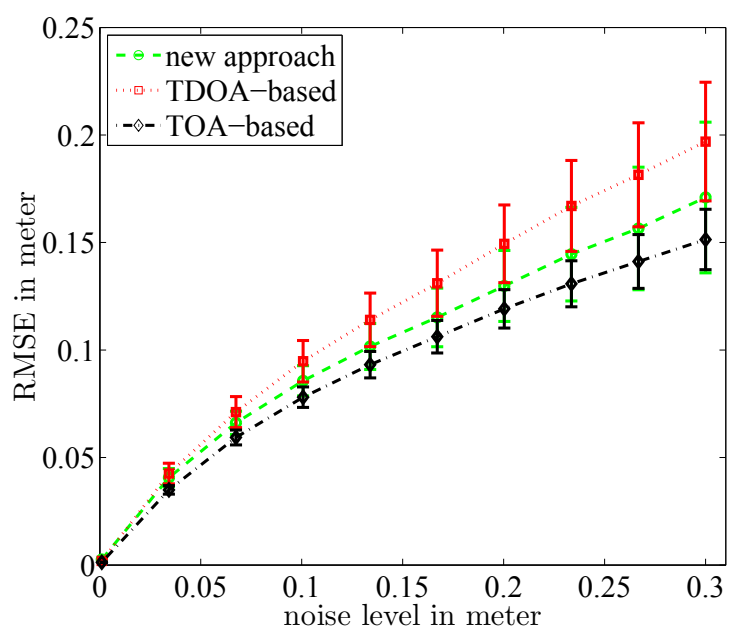

(a) Mean and standard deviation of the RMSE for the three approaches. The black line is the result, when the offset is exactly known. The green line show the result of the proposed approach. The result of the TDOA-based approach is shown in the red line.

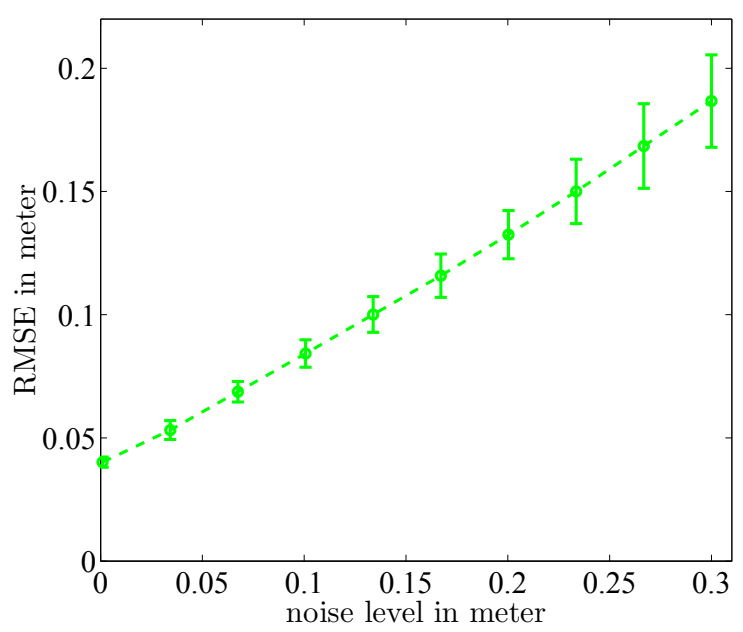

(b) Mean and standard deviation of the RMSE for the offset.

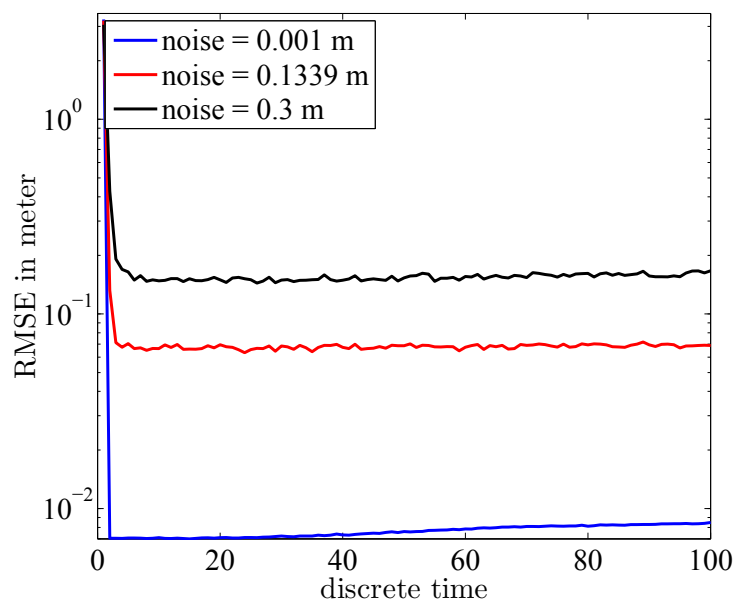

(c) Mean of the RMSE of the Offset at different noise level for all trajectories.

Figure 6: Results when the minimal number of sensors is used. 
In the first simulation results, the average of the RMSE of the new approach has a maximum deviation of 0.0024 meter compared to the TOA-based approach. The maximum deviation by using the TDOA-based approach is 0.0263 meter. In the case of the minimum number of sensors, the maximum deviation increases for the new approach to 0.0195 meter and for the TDOAbased approach to 0.0455 meter. This shows that the proposed approach determines the offset and updates the state accurately compared to the TDOA-based approach and the TOA-based approach, where the TOAbased approach can be seen as the lower bound for this kind of problem.

\section{Conclusions}

In this paper, a two-step procedure for multilateration tracking is presented, where the measured receiving times are directly used for estimating the position. The proposed approach relies on optimal stochastic linearization for calculating the range offset and updating the position. Based on analytic expressions for the moments, the offset is calculated by minimizing the Mahalanobis distance. In a second step, this result is used for updating the state by using Bayesian inference. Hence, no sample-based methods have to been applied, because the required moments are calculated in closed form. Furthermore, the computational demand is lower compared to sample-based methods, because for example no matrix roots have to be calculated.

A disadvantage of this procedure is that no uncertainty quantity can be given for the estimated offset compare to approaches, where the offset is augmented in the state vector. However, in some scenarios state augmentation is not feasible, when the offset cannot be described by an adequate system model. Hence, the advantage of the proposed approach is that no assumptions about the dynamic behavior for the offset has to be made.

In the multilateration tracking scenario, the new approach provides better estimation results than the TDOA-based approach, even for higher noise levels. Compared to the TDOA-based approach, where hyperboloids are intersected, the new approach relies on the intersection of spheres depending on the estimate of the offset. Hence, the new approach can be interpreted as the intersection of spherical hypercones.

\section{References}

[1] Y. Bar-Shalom, X.-R. Li, and T. Kirubarajan. Estimation with Applications to Tracking and Navigation: Theory, Algorithms, and Software. John Wiley \& Sons, Inc., New York, NY, USA, 2001.

[2] M. S. Brandstein, J. E. Adcock, and H. F. Silverman. A Closed-Form Location Estimator for Use with Room Environment Microphone Arrays.
IEEE Transactions on Speech and Audio Processing, 5(1):45-50, January 1997.

[3] Y. Chan and K. Ho. A Simple and Efficient Estimator for Hyperbolic Location. IEEE Transactions on Signal Processing, 42(8):1905-1915, August 1994.

[4] M. Clark and R. Vinter. A New Class of Moment Matching Filters for Nonlinear Tracking and Estimation Problems. In 2006 IEEE Nonlinear Statistical Signal Processing Workshop, pages 108-112, Sept. 2006.

[5] M. F. Huber and U. D. Hanebeck. Gaussian Filter based on Deterministic Sampling for High Quality Nonlinear Estimation. In Proceedings of the 17th IFAC World Congress (IFAC 2008), volume 17, Seoul, Korea, July 2008.

[6] K. Ito and K. Xiong. Gaussian Filters for Nonlinear Filtering Problems. IEEE Transactions on Automatic Control, 45(5):910-927, May 2000.

[7] S. J. Julier and J. K. Uhlmann. Unscented Filtering and Nonlinear Estimation. Proceedings of the IEEE, 92(3):401-422, 2004.

[8] M. Norgaard, N. Poulsen, and O. Ravn. New Developments in State Estimation for Nonlinear Systems. Automatica, 36(11):1627-1638, Nov. 2000.

[9] A. Papoulis and S. U. Pillai. Probability, Random Variables and Stochastic Processes. McGraw-Hill Science/Engineering/Math; 4. Edition, 2002.

[10] C. Savage, R. Cramer, and H. Schmitt. TDOA Geolocation with the Unscented Kalman Filter. In Proceedings of the 2006 IEEE International Conference on Networking, Sensing and Control, 2006 (ICNSC '06), pages 602-606, 2006.

[11] R. O. Schmidt. A new Approach to Geometry of Range Difference Location. IEEE Transaction on Aerospace and Electronic Systems, AES-8(6):821835,1972 .

[12] J. O. Smith and J. S. Abel. Closed-Form LeastSquares Source Location Estimation from RangeDifference Measurements. IEEE Transactions of Acoustics, Speech, and Signal Processing, ASSP35(12):1661-1669, Dezember 1987.

[13] G. Welch, B. D. Allen, A. Ilie, and G. Bishop. Measurement Sample Time Optimization for Human Motion Tracking/Capture Systems. In Proceedings of Trends and Issues in Tracking for Virtual Environments, Workshop at the IEEE Virtual Reality 2007 Conference, 2007. 